\title{
Anaphylaxie auch bei topischer Arzneimittelapplikation
}

\section{Sogar bei nicht mukosaler topischer Anwendung von Arzneimitteln kann es zu anaphylaktischen Reaktionen kommen. Das zeigen bundesweite Daten über unerwünschte Arzneimittelwirkungen sowie die internationale Literatur.}

\begin{abstract}
A naphylaxie und toxische epidermale Nekrolyse oder Stevens-JohnsonSyndrom sind Maximalvarianten unerwünschter Wirkungen einer systemischen Medikamentengabe. Ob solche Arzneimittelreaktionen auch nach nicht mukosaler, topischer Applikation auftreten, wurde in einer umfangreichen Datenbank- und Literaturrecherche überprüft, berichtete Priv.-Doz. Dr. Bernhardt Sachs, Bundesinstitut für Arzneimittel und Medizinprodukte (BfArM), Bonn.

Um mögliche Fälle zu identifizieren, wurde zunächst in der BfArM-Datenbank recherchiert, die einen Bestand von rund 350.000 Berichten über unerwünschte Arzneimittelwirkungen in Deutschland umfasst. Als weitere Quelle wurde die internationale Literatur herangezogen. Nach Evaluierung der identifizierten Fälle konnten aus der Datenbank 28 Fälle und aus der Literatur 48 Fälle mit anaphylaktischen Arzneimittelreaktionen einer weiteren Analyse unterzogen werden. Bei den inkriminierten Arzneimitteln handelte es sich bei neun der 28 Datenbankfälle um hyperämisierende Substanzen, in vier Fällen um Antibiotika (Aminoglykoside) und in weiteren vier Fällen um Antiseptika. Von den in der Literatur berichteten Fällen konnte fast die Hälfte auf Antibiotika und ein Viertel auf verschiedene Antiseptika zurückgeführt werden.

Als Risikofaktor erwies sich der Hautzustand: Bei 32 der insgesamt 76 Fälle war vermerkt, dass die Applikation des ursächlichen Medikaments auf Hautläsionen wie Ulzera erfolgt war, bei weiteren 20 war die Barrierefunktion der Haut eingeschränkt gewesen. Nur bei einem der Patienten wurde die Haut explizit als gesund eingeschätzt, in allen übrigen Fällen fehlten Angaben zum Hautzustand. Als weiterer Risikofaktor wurden Allergien eruiert: Bei
\end{abstract}

Eukalyptusöl, Kiefernnadelöl und Menthol enthielt. Ein kausaler Zusammenhang mit dem Stevens-Johnson-Syndrom wurde für alle drei Arzneimittel gleichrangig als möglich beurteilt. Bei dem zweiten Fall handelte es sich um eine Patientin mit Mycosis fungoides, die topisch mit Mechlorethamin behandelt wurde. Risikofaktor schien hier eine erhöhte Resorption über die Hautläsionen zu sein.

Anaphylaxien sind also auch nach nicht mukosaler topischer Arzneimittelapplikation möglich, während mit toxischer epidermaler Nekrolyse und Stevens-Johnson-Syndrom kaum zu rechnen ist, fasste Sachs die Ergebnisse zusammen. Bei bekannten Allergien könne durch das Mitführen eines Allergiepasses das Risiko für eine Anaphylaxie verringert werden.

abd

Sachs B, Fischer-Barth W, Merk H. Anaphylaxis and toxic epidermal necrolysis or Stevens-Johnson syndrome after nonmucosal topical drug application: fact or fiction? 19. Mainzer Allergie-Workshop, Mainz, 16.-17. März 2007 